\title{
Effects of Parecoxib on Pain Threshold and Inflammatory Factors IL-1 $\beta$, IL-6 and TNF- $\alpha$ in Spinal Cord of Rats with Bone Cancer Pain
}

\author{
Jun Chen, Xiufeng Cong, Xiuzhu Zhan, Zheng Zhou and Wei Zheng
}

\begin{abstract}

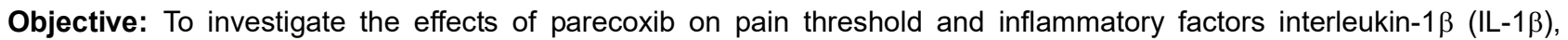
interleukin-6 (IL-6) and tumor necrosis factor- $\alpha$ (TNF- $\alpha$ ) in spinal cord of rats with bone cancer pain.

Study Design: An experimental study.

Place and Duration of Study: Department of Oncology, Shengjing Hospital of China Medical University, China, from March 2017 to May 2018.

Methodology: Twenty-four healthy female Sprague-Dawley rats were selected and the bone cancer pain model was inoculated with W256 breast cancer cell into the bone marrow. Rats with bone cancer pain were randomly divided into the model group and the parecoxib group on the $7^{\text {th }}$ day postoperation, with 12 rats in each group. Another 12 rats were taken as the control group. Rats in the parecoxib group were given intraperitoneal injection of parecoxib $(8 \mathrm{mg} / \mathrm{kg})$ for 10 consecutive days since the 15th day after operation. Mechanical pain threshold, thermal pain threshold, and the levels of inflammatory factors IL-1 $\beta$, IL- 6 and TNF- $\alpha$ in spinal cord of rats in each group were compared.

Results: On the 14th postoperative day, mechanical pain threshold and thermal pain threshold of rats in the model group and the parecoxib group were significantly decreased compared with those in the control group $(p<0.001)$. After 5 and 10 days of administration, mechanical and thermal pain threshold of rats in the parecoxib group were significantly higher than those in the model group and the control group ( $p<0.001$ ). After 10 days of administration, levels of IL-1 $\beta$, IL- 6 and TNF- $\alpha$ in spinal cord of the model group were higher than those of the control group $(p<0.001)$; and levels of IL-1 $\beta$, IL- 6 and TNF- $\alpha$ in spinal cord of the parecoxib group were significantly lower than those in the model group and the control group $(p<0.001)$.

Conclusion: Parecoxib can alleviate hyperalgesia in rats with bone cancer pain, increase pain threshold and inhibit the up-regulation of inflammatory factors in the spinal cord. Parecoxib may achieve analgesic effects by down-regulating the expression of IL-1 $\beta$, IL-6 and TNF- $\alpha$ in the spinal cord.
\end{abstract}

Key Words: Parecoxib, Bone cancer pain, Rat, Analgesia, IL-1 $\beta$, IL-6, TNF- $\alpha$.

\section{INTRODUCTION}

Bone cancer pain is a complex pain state consisting of basal pain, spontaneous pain, and event pain, or exercise-induced pain.1,2 Allodynia refers to a stimulus, which does not cause pain in normal physiological conditions such as a light touch, which causes a pain response. Hyperalgesia refers to a stimulus, which causes pain in a normal state, and produces more intense pain. The spinal dorsal root ganglia (DRG) are the primary centre for the transmission and handling of pain signals as well as the first-level neuron for pain transmission. ${ }^{3}$ Various inflammatory cells and immune cells in tumor tissues secrete various cytokines, which act on their respective receptors, activating and sensitising peripheral receptors (i.e., peripheral sensitisation).

Department Of Oncology, Shengjing Hospital of China

Medical University, Shenyang, 110000, China

Correspondence: Dr. Wei Zheng, Department Of Oncology,

Shengjing Hospital of China Medical University, Shenyang,

110000, China

E-mail: yvahc2@163.com

Received: September 06, 2018; Accepted: November 19, 2018
Moreover, persistent excitatory impulses of peripheral sensitisation are afferent into the dorsal horn of the spinal cord through DRG $A \delta$ and C-fibers, leading to increased excitability of central pain relay cell and increased sensitivity (i.e., central sensitisation), thus causing the cancer pain. ${ }^{4}$ Related data show that inflammatory factors such as interleukin-1 $\beta$ (IL-1 $\beta$ ), interleukin-6 (IL-6) and tumor necrosis factor- $\alpha$ (TNF- $\alpha$ ) in the spinal cord are involved in the occurrence and development of bone cancer pain. ${ }^{5}$

Parecoxib is a prodrug of valdecoxib and belongs to a new generation of selective COX-2 inhibitors. It is currently widely used as an analgesic in clinical practice. Recent studies suggested that parecoxib might have a valuable place in the management of cancer pain. ${ }^{6} \mathrm{~A}$ study concluded that short-term daily subcutaneous parecoxib injection was effective for malignant bone pain when added to existing medication. ${ }^{7}$ However, very little is known about the mechanism of parecoxib in the treatment of bone cancer pain.

The objective of this study was to investigate the effects of parecoxib on the pain threshold and the expression of inflammatory cytokines IL-1 $\beta$, IL- 6 and TNF- $\alpha$ in rats 
with bone cancer pain, so as to reveal the mechanism of parecoxib on bone cancer pain.

\section{METHODOLOGY}

This study was conducted at the Department of Oncology, Shengjing Hospital of China Medical University, China, from March 2017 to May 2018, after approval from the Hospital Ethical and Research Committee. Thirty-six healthy female Sprague-Dawley rats were selected, with a body mass of $155-178 \mathrm{~g}$, (averaged $165.34 \pm 3.72 \mathrm{~g}$ ).

Then 24 rats were randomly selected for anesthesia, the upper skin of their left hind limb was cut into a small incision of about $1.0 \mathrm{~cm}$, and the tibia was exposed. A 7-gauge needle was rotated and pierced at the upper $1 / 3$ of the tibia and into the marrow cavity for a hole. A 5 - $\mu \mathrm{l}$ micro-syringe was used to extract $3 \mu \mathrm{l}$ of about $4 \times 10^{3}$ W256 breast cancer cell suspension into the bone marrow cavity. The pinhole was immediately sealed with bone wax. The incision was sutured and a little gentamicin solution was dropped to prevent infection. After operation, the rats were routinely reared for seven days. After the use of $3 \%$ pentobarbital sodium for anesthesia, the limbs were observed under a highfrequency mammography machine. As the upper end of the tibia showed obvious defects, the rat model with bone cancer pain was successfully established. On the 7 th day postoperation, the 24 rats with bone cancer pain were randomly divided into the model group and the parecoxib group, with 12 in each group. Another 12 rats were injected with physiological saline instead of W256 breast cancer cell suspension into the bone marrow cavity as the control group. Rats in each group were given medicine on the 15th day after operation, twice a day for 10 days. Rats in the parecoxib group were given intraperitoneal injection of parecoxib $(8 \mathrm{mg} / \mathrm{kg})$, and rats in the control group and the model group were given an equal amount of physiological saline by intraperitoneal injection.

On the $7^{\text {th }}$ and $14^{\text {th }}$ days postoperation, 5th day after administration ( $20^{\text {th }}$ day after operation) and 10 th day after administration ( $25^{\text {th }}$ day after operation), the changes of mechanical pain threshold in each group were measured by toe-tenderness method and the changes of thermal pain threshold were measured by hot plate method.

On the $10^{\text {th }}$ day after administration $\left(25^{\text {th }}\right.$ day after operation), rats in each group were measured with mechanical and thermal pain threshold. The rats were deeply anesthetised by intraperitoneal injection of $4 \%$ chloral hydrate $(400 \mathrm{mg} / \mathrm{kg})$, and the left side of L4-L6 spinal dorsal horn segment was quickly removed, weighed and added with the lysate at a ratio of $200 \mu \mathrm{L}$ of tissue lysate to $100 \mathrm{mg}$ of spinal cord tissue, and well homogenised on ice. The supernatant was collected after centrifugation and the levels of inflammatory factors IL-1 $\beta$, IL- 6 and TNF- $\alpha$ in the spinal cord tissues of each group were detected by enzyme-linked immunosorbent assay (ELISA).

Data was entered and analysed by using SPSS version 25.0. Continuous data was described as mean $\pm S D$. One-way analysis of variance was used for comparison among the three groups. SNK method was used for comparison between two groups. P-value of less than 0.05 was considered significant.

\section{RESULTS}

On the $7^{\text {th }}$ day postoperation, there was no significant difference in mechanical pain threshold among the control group, the model group, and the parecoxib group $(p=0.213)$. On the 14th day postoperation, mechanical pain threshold of the model group and the parecoxib group was significantly decreased compared with that of the control group $(p<0.001)$. After 5 and 10 days of administration, mechanical pain threshold of the parecoxib group was significantly higher than that of the model group and the control group $(p<0.001$, Table I).

On the $7^{\text {th }}$ day postoperation, there was no significant difference in thermal pain threshold among the control group, the model group and the parecoxib group $(p=0.982)$. On the $14^{\text {th }}$ day postoperation, thermal pain threshold of the model group and the parecoxib group was significantly lower than that of the control group $(p<0.001)$. After 5 and 10 days of administration, the thermal pain threshold of the parecoxib group was

Table I: Comparison of mechanical pain thresholds at different time points (unit: kg).

\begin{tabular}{|c|c|c|c|c|c|c|c|c|c|}
\hline \multirow[t]{2}{*}{ Groups } & \multirow[t]{2}{*}{ Number of mice } & \multicolumn{2}{|c|}{7 days after operation } & \multicolumn{2}{|c|}{14 days after operation } & \multicolumn{2}{|c|}{5 days after administration } & \multicolumn{2}{|c|}{10 days after administration } \\
\hline & & Mean $\pm S D$ & $p$-value & Mean $\pm S D$ & $p$-value & Mean $\pm S D$ & $p$-value & Mean $\pm S D$ & $p$-value \\
\hline Control group & 12 & $0.291 \pm 0.018$ & 0.213 & $0.304 \pm 0.027$ & $<0.001$ & $0.310 \pm 0.031$ & $<0.001$ & $0.313 \pm 0.044$ & $<0.001$ \\
\hline Model group & 12 & $0.278 \pm 0.016$ & & $0.248 \pm 0.028$ & & $0.226 \pm 0.027$ & & $0.231 \pm 0.033$ & \\
\hline Parecoxib group & 12 & $0.283 \pm 0.020$ & & $0.259 \pm 0.023$ & & $0.408 \pm 0.043$ & & $0.394 \pm 0.049$ & \\
\hline
\end{tabular}

Table II: Comparison of thermal pain thresholds at different time points (unit: s).

\begin{tabular}{|c|c|c|c|c|c|c|c|c|c|}
\hline \multirow[t]{2}{*}{ Groups } & \multirow[t]{2}{*}{ Number of mice } & \multicolumn{2}{|c|}{7 days after operation } & \multicolumn{2}{|c|}{14 days after operation } & \multicolumn{2}{|c|}{5 days after administration } & \multicolumn{2}{|c|}{10 days after administration } \\
\hline & & Mean \pm SD & p-value & Mean $\pm S D$ & $p$-value & Mean \pm SD & $p$-value & Mean $\pm S D$ & $p$-value \\
\hline Control group & 12 & $18.87 \pm 4.82$ & 0.982 & $18.95 \pm 4.25$ & $<0.001$ & $19.36 \pm 4.21$ & $<0.001$ & $19.77 \pm 4.33$ & $<0.001$ \\
\hline Model group & 12 & $18.53 \pm 4.16$ & & $11.27 \pm 3.87$ & & $11.94 \pm 3.01$ & & $11.52 \pm 3.61$ & \\
\hline Parecoxib group & 12 & $18.74 \pm 3.72$ & & $11.52 \pm 3.66$ & & $27.18 \pm 7.95$ & & $26.63 \pm 5.16$ & \\
\hline
\end{tabular}


Table III: Comparison of IL-1 $\beta$, IL-6 and TNF- $\alpha$ levels in spinal cord of rats in each group after 10 days of administration.

\begin{tabular}{l|c|cc|cc|c}
\hline Groups & $\mathrm{n}$ & \multicolumn{2}{|c|}{$\mathrm{IL}-1 \beta(\mathrm{pg} / \mathrm{ml})$} & \multicolumn{2}{|c|}{$\mathrm{IL}-6(\mathrm{pg} / \mathrm{ml})$} & \multicolumn{2}{c}{ TNF- $\alpha(\mathrm{pg} / \mathrm{ml})$} \\
\cline { 2 - 6 } & & Mean \pm SD & $\mathrm{p}$-value & Mean \pm SD & $\mathrm{p}$-value & Mean \pm SD \\
\hline Control group & 12 & $80.56 \pm 17.22$ & $<0.001$ & $383.07 \pm 74.84$ & $<0.001$ & $89.96 \pm 6.23$ \\
\hline Model group & 12 & $261.24 \pm 81.63$ & & $867.32 \pm 93.41$ & & $124.23 \pm 9.38$ \\
\hline Parecoxib group & 12 & $152.73 \pm 35.57$ & $554.29 \pm 60.47$ & 0.001 \\
\hline
\end{tabular}

significantly higher than that of the model group and the control group $(p<0.001$, Table II).

After administration for 10 days, the levels of IL-1 $\beta$, IL- 6 and TNF- $\alpha$ in the spinal cord of the control group, the model group and the parecoxib group were significantly different $(p<0.001)$; levels of IL-1 $\beta, \mathrm{IL}-6$ and TNF- $\alpha$ in spinal cord of the model group were higher than those of the control group ( $p<0.001$ ); and levels of IL-1 $\beta$, IL-6 and TNF- $\alpha$ in spinal cord of the parecoxib group were significantly lower than those in the model group and the control group $(p<0.001$, Table III).

\section{DISCUSSION}

Parecoxib is the first selective cyclooxygenase-2 (COX-2) inhibitor that can be injected intravenously and intramuscularly. It is now widely used for the prevention and treatment of moderate to severe pain. ${ }^{8}$ Studies have shown that parecoxib can reduce the need for opioid analgesics in surgical patients. Moreover, $40 \mathrm{mg}$ parecoxib is more effective than $4 \mathrm{mg}$ morphine in the treatment of postoperative pain. ${ }^{9}$ Parecoxib is recommended for the treatment of cancer pain. ${ }^{10}$ However, the mechanism of action of parecoxib against cancer pain is still not clear.

In this study, a bone cancer pain model was successfully prepared by injecting high-erosive W256 breast cancer cells into the sacral bone marrow of the studied rats. There are many advantages of using W256 breast cancer cells to establish a bone cancer pain model. First, it exhibits pain behaviour similar to human bone cancer pain. Second, the pain level measurement index is easy to detect and the quantification is more accurate. Third, the success rate of the model preparation is higher. Finally, the preparation process is relatively easy, so it is easy to practice. 11 It is ideal to carry out experimental study 6-18 days after the establishment of rat model of bone cancer pain. In this experiment, rats were given medicine 15 days after the establishment of the bone cancer pain model, which is in line with the reasonable time window of bone cancer pain research. ${ }^{12}$ Through this experiment, it was observed that 5 and 10 days after the model group, rats were given intraperitoneal injection of parecoxib, the mechanical and thermal hyperalgesia in rats can be alleviated. The mechanical and thermal pain threshold of rats with bone cancer pain significantly improved, suggesting that parecoxib can alleviate hyperalgesia in rats with bone cancer pain and improve their pain threshold. This conclusion is basically consistent with the findings of others. ${ }^{13}$
In chronic pain, glial cells can mediate the release of various pro-inflammatory factors. These factors, represented by IL-1 $\beta$, IL- 6 and TNF- $\alpha$, play an important role in the development of chronic pain. 14,15 Noxious stimulation can cause up-regulation of IL-1 $\beta$, IL- 6 and TNF- $\alpha$ expression. IL-1 $\beta$ can induce the expression of various inflammatory pain-causing substances such as IL-6, TNF- $\alpha$, etc.; and its degree of elevation is closely related to pain. ${ }^{16,17} \mathrm{IL}-6$ has a pain-inducing effect on the peripheral or central nervous system and stimulates the massive release of COX-2.18 Previous studies have found that inflammatory factors such as IL-1 $\beta$, IL- 6 and TNF- $\alpha$ in the spinal and dorsal ganglia of animal models participate in and mediate the occurrence of bone cancer pain. ${ }^{19}$ Inflammatory factors, such as IL-1 $\beta$, IL-6 and TNF- $\alpha$ may affect and amplify the spinal cord's perception of noxious stimuli, eventually leading to hyperalgesia, which plays an important role in the occurrence and maintenance of pain sensitivity in patients with bone cancer pain. ${ }^{20}$ The experiments showed that the levels of IL- $1 \beta, \mathrm{IL}-6$ and TNF- $\alpha$ in the spinal cord of the model group were higher than those of the control group after 10 days of administration. The levels of IL-1 $1 \beta$, IL- 6 and TNF- $\alpha$ in the spinal cord of the parecoxib group were significantly lower than those of the model group and the control group. This indicates that parecoxib inhibits the secretion and release of inflammatory factors in the spinal cord of rats with bone cancer pain, thereby reducing the activation and sensitisation of primary afferent neurons, and finally reducing the cancer pain.

The authors believe that parecoxib has a good analgesic effect on bone cancer pain. It is speculated that parecoxib may have an analgesic effect by eliminating inflammation and reducing inflammatory swelling. It is worth mentioning that because bone cancer pain is the result of a combination of factors, the analgesic effect of parecoxib on bone cancer pain may also involve other mechanisms. In addition, the results of animal models are still far from those of the human, so the analgesic mechanism of parecoxib remains to be further studied.

\section{CONCLUSION}

Parecoxib can alleviate hyperalgesia in rats with bone cancer pain, increase the pain threshold and inhibit the up-regulation of inflammatory factors in the spinal cord. Parecoxib may achieve analgesic effects by downregulating the expression of IL-1 $\beta$, IL- 6 and TNF- $\alpha$ in the spinal cord. 


\section{REFERENCES}

1. Christian-Miller N, Frenette C. Hepatocellular cancer pain: Impact and management challenges. $J$ Hepatocell Carcinoma 2018; 5:75-80.

2. Edwards KA, Havelin JJ, Mcintosh MI, Ciccone HA, Pangilinan K, Imbert I, et al. A kappa opioid receptor agonist blocks bone cancer pain without altering bone loss, tumor size, or cancer cell proliferation in a mouse model of cancer-induced bone pain. J Pain 2018; 19:612-25.

3. Jin SX, Zhuang ZY, Woolf CJ, Ji RR. p38 mitogen-activated protein kinase is activated after a spinal nerve ligation in spinal cord microglia and dorsal root ganglion neurons and contributes to the generation of neuropathic pain. $J$ Neurosci 2003; 23:4017-22.

4. Ikoma M, Kohno T, Baba H. Differential presynaptic effects of opioid agonists on Adelta- and C-afferent glutamatergic transmission to the spinal dorsal horn. Anesthesiology 2007; 107:807-12.

5. Ren BX, Ji Y, Tang JC, Sun DP, Hui X, Yang DQ, et al. Effect of tanshinone iia intrathecal injections on pain and spinal inflammation in mice with bone tumors. Genet Mol Res 2015; 14:2133-8.

6. Armstrong P, Wilkinson P, McCorry NK. Use of parecoxib by continuous subcutaneous infusion for cancer pain in a hospice population. BMJ Support Palliat Care 2018; 8:25-9.

7. Kenner DJ, Bhagat S, Fullerton SL. Daily subcutaneous parecoxib injection for cancer pain: An open label pilot study. J Palliat Med 2015; 18:366-72.

8. Luo X, Lv F, Peng M. Analgesic effect of different dosage of flurbiprofen axetil in laparoscopic cholecystectomy in comparison with other analgesic drugs. Pak J Pharm Sci 2017; 30:1895-8.

9. Rasmussen GL, Steckner K, Hogue C, Torri S, Hubbard RC. Intravenous parecoxib sodium foracute pain after orthopedic knee surgery. Am J Orthop (Belle Mead NJ) 2002; 31:336-43.

10. Jin X, Zhou F, Liu Y, Cheng C, Yao L, Jia Y, et al. Simultaneous determination of parecoxib and its main metabolites valdecoxib and hydroxylated valdecoxib in mouse plasma with a sensitive LC-MS/MS method to elucidate the decreased drug meta- bolism of tumor bearing mice. J Pharm and Biomed Anal 2018; 158:1-7.

11. Wojtukiewicz MZ, Hempel D, Sierko E, Tucker SC, Honn KV. Protease-activated receptors (PARs) - biology and role in cancer invasion and metastasis. Cancer Metastasis Rev 2015; 34:775-96.

12. Yao M, Chang XY, Chu YX, Yang JP, Wang LN, Cao HQ, et al. Antiallodynic effects of propentofylline elicited by interrupting spinal glial function in a rat model of bone cancer pain. J Neurosci Res 2011; 89:1877-86.

13. Cui $W$, Yu X, Zhang $H$. The study of different approaches of parecoxib sodium pretreatment on the behaviour of rats with neuropathic pain. Cell Biochem Biophys 2015; 72:137-40.

14. Capossela S, Pavlicek D, Bertolo A, Landmann G, Stoyanov JV. Unexpectedly decreased plasma cytokines in patients with chronic back pain. J Pain Res 2018; 11:1191-8.

15. Richards GC, Lluka LJ, Smith MT, Haslam C, Moore B, O'Callaghan $\mathrm{J}$, et al. Effects of long-term opioid analgesics on cognitive performance and plasma cytokine concentrations in patients with chronic low back pain: a cross-sectional pilot study. Pain Rep 2018; 3:e669.

16. Al-Mazidi S, Alotaibi M, Nedjadi T, Chaudhary A, Alzoghaibi M, Djouhri L. Blocking of cytokines signalling attenuates evoked and spontaneous neuropathic pain behaviours in the paclitaxel rat model of chemotherapy-induced neuropathy. Eur $J$ Pain 2017; 22:810-21.

17. Rocha LW, Sonza DR, Broering MF, Nunes R, de CamposBuzzi F, Corrêa R, et al. Synthetic chalcones as potential tool for acute- and chronic-pain control. Biomed Pharmacother 2018; 104:437-50.

18. Zanjani TM, Sabetkasaei M, Karimian B, Labibi F, Farokhi B, Mossafa $\mathrm{N}$. The attenuation of pain behaviour and serum interleukin- 6 concentration by nimesulide in a rat model of neuropathic pain. Scand J Pain 2010; 1:229-34.

19. Zhao J, Zhang H, Liu SB, Han P, Hu S, Li Q, et al. Spinal interleukin-33 and its receptor ST2 contribute to bone cancerinduced pain in mice. Neuroscience 2013; 253:172-82.

20. Hao W, Chen L, Wu LF, Yang F, Niu JX, Kaye AD, et al. Tanshinone iia exerts an antinociceptive effect in rats with cancer-induced bone pain. Pain Physician 2016; 19:465-76. 